\title{
Efficacy of combination therapy of Hyaluronic acid and Methylprednisolon verses Hyaluronic acid single therapy in knee Osteoarthritis : A comparative study
}

\author{
S A Ullah ${ }^{1}$, F Ahmed ${ }^{2}$, N Sultana ${ }^{3}$, S A Hossain ${ }^{4}$
}

1 Dr. Sk. Ashraf Ullah Associate Professor (C.C.) Dept of Orthopaedics Northern International Medical College, Dhaka

2

Dr. Fakhruddin Ahmed Junior consultant U.H.C. Rupganj, Narayanganj

3

3 Dr. Nasreen Sultana Junior consultant U.H.C. Rupganj, Narayanganj

${ }^{4}$ Prof. Dr. Sk. Akbar Hossain Professor and Head Dept. of Community Medicine Northern International medical College, Dhaka

Correspondence

Dr. Sk. Ashraf Ullah

Associate Professor (C.C.)

Dept of Orthopaedics

Northern International Medical

College, Dhaka

E-mail: drashraf158@gmail.com

\section{Abstract}

Background : Osteoarthritis of knee joint is one of the most common problem which cause pain, loss of function even joint disability and increases misery and morbidity in old age. Now a day's intra-articular injection of single Hyaluronic acid or corticosteroids (Methylprednisolone) are using for knee osteoarthritis cases. But the combined therapy by injection of both Hyaluronic acid and Methyl prednisolone are not so much practiced and still under debate.

Objectives : To compare the efficacy of intra-articular injection of combined Hyaluronic acid and Methyl prednisolone with single Hyaluronic acid alone.

Methods : A prospective, comparative study was done in Northern International Medical College Hospital, Dhaka, during May 2017 to April 2018 at Out Patient Department with 80 patients (132 knees). Patients were randomized into two groups: Group-A with 40 patients with 15 unilateral and 25 bilateral knee cases (65 knees) were treated with combined Hyaluronic acid and Methyl prednisolone and Group-B with 40 patients with13 unilateral and 27 bilateral knee cases (67 knees) were infiltrated with single Hyaluronic acid. Results were evaluated with Knee Society Score (KSS) and Visual analogue score (VAS) at the 1st week, 1st month and 3rd month after injection.

Result : In both Group (A and B) patients of age $61-70$ years were highest, $50 \%$ and $45 \%$ respectively. Male and female ratio was 1:2.2. At the 1st week KSS for pain in Group-A (mean 65.6) was better than Group-B (mean 57.4). At the 1st month mean pain scores (Group-A 72.4, Group-B 64.4) and the 3rd month (Group-A 81.4, Group-B 75.6) and mean function scores at 1st week (Group-A 61.3, Group-B 49.6)), 1st month (Group-A65.1, Group-B 53.6) and 3rd month (Group-A68.4, Group-B 59.4). The KSS for pain and function improved in combination therapy group.VAS decreased in both groups.

Conclusion : As combination therapy by Hyaluronic acid and Methyl prednisolone showed better efficacy than single Hyaluronic acid. Therefore, combination of these two drugs in a single vial can be used as an effective and easy nonoperative treatment of knee osteoarthritis.

Key words : Osteoarthritis, Hyaluronic acid, Methyl prednisolone, Knee Society Score (KSS), Visual analogue score (VAS).

DOI: https://doi.org/10.3329/nimcj.v10i2.45429

Northern International Medical College Journal Vol. 10 No. 2 January 2019, Page 366-369

\section{Introduction}

Osteoarthritis, the most common form of arthritis, is a progressive degenerative disease characterized by gradual loss of joint cartilage with local inflammation resulting in pain and loss of movement. ${ }^{1}$ It is a common disorder whose prevalence increases with age. From 35 years of age $50 \%$ of people present some sign of this disease. This figure rises to $80 \%$ after 55 years and $85 \%$ from 65 years of age. The prevalence of symptomatic knee osteoarthritis was $21.2 \%$ overall: $10.7 \%$ in men and $26.7 \%$ in women. ${ }^{2}$

Osteoarthritis has a multifactorial etiology and can be primary (with no obvious cause) or secondary (due to trauma, infiltrative disease or connective tissue disease). Many mechanical and chemical factors such as-obesity, joint injuries, older age, bone deformity, heredity etc. have been suggested as the responsible cause of cartilage destruction leading to osteoarthritis. ${ }^{3}$

The pathogenesis of osteoarthritis involves a degradation of cartilage and remodeling of bone due to an active response of chondrocytes in the articular cartilage and the inflammatory cells in the surrounding tissues. The release of enzymes from these cells breaks down cartilage and 
proteoglycans destroying the articular cartilage. ${ }^{4}$

Clinical features include pain and stiffness in joints, worsened with activity. ${ }^{3}$ Early diagnosis and treatment is essential for reducing the morbidity of knee Osteoarthritis. Treatment modality includes-weight reduction, Exercise, Analgesics, tropical analgesics, Non Steroid Anti Inflammatory Drugs (NSAID)s, Physical therapy, Injection (Steroid, Hyaluronic acid) and Surgery. Many types of treatment options are available for osteoarthritis.

Methyl prednisolone as a steroid when injected into a painful area (such as a joint or muscle) can reduce the inflammation in that area, relieving pain, reducing tissue swelling and improving function and mobility ${ }^{5}$ Hyaluronic acid (HA)- is a natural compound present in healthy synovial fluid. When Hyaluronic acid is injected in osteoarthritic joint, it provides hyaluronan as lubricant and helps in decreasing joint pain and inflammation. ${ }^{6}$

Although different clinical trials have been done by using intra articular individual drug but there are no strong trails available on combination therapy. Combined use of Hyaluronic acid and Methyl prednisolone can be an effective treatment option in case of knee osteoarthritis especially when these two drugs can make available in a single vial. Keeping this view in mind the present study was an attempt to evaluate the efficacy of combination therapy (HA and steroid) verses single therapy (HA) was done.

\section{Methods}

This prospective, comparative study was conducted in Northern International Medical College Hospital, Bangladesh from May 2017 to April 2018 at Orthopaedic outpatient department (OPD). Initially 130 patients were selected for the present study. After fulfilling the inclusion and exclusion criteria, 80 patients were finally enrolled.

Inclusion criteria : Primary osteoarthritis of knee according to Kellgren Lawrence Grade II and III who had symptoms for more than 6 months, age between 41 and 70 years.

Exclusion criteria : Patients with secondary osteoarthritis, diabetes mellitus, thyroid problem, valgus or varus deformity, flexion deformity, and past history of treatment with intraarticular injection.

Among 80 patients total 132 knees where 28 were unilateral knee case and 52 bilateral knee cases were studied. Bilateral knee pain case was considered as single patient. The patients were randomized into 2 groups:

Group-A (40 patients; 65 knees- 15 unilateral and 25 bilateral): received combination therapy by intra-articular injection of $4 \mathrm{ml}$ $(10 \mathrm{mg} / \mathrm{ml})$ of high molecular weight Hyaluronic acid (Hyalgan) with $1 \mathrm{ml}(40 \mathrm{mg} / \mathrm{ml})$ of Methyl prednisolone (Solumed) as a single combined dose.
Group-B (40 patients; 67 knees-13 unilateral and 27 bilateral): received single intra-articular injection of $4 \mathrm{ml}(10 \mathrm{mg} / \mathrm{ml})$ of high molecular weight of Hyaluronic acid.

All the patients were given injection via infero-lateral Para patellar approach. They were advised to attend orthopedic OPD on scheduled date for follow up. Knee society score for pain (KSS pain), knee society score for function (KSS function) and visual analogue scale (VAS) score were evaluated just before the injection and then after injection at the 1st week, 1st month and the 3rd month. The use of NSAIDs or others treatment was stopped throughout the study period.

After obtaining ethical permission and taking informed consent data were collected on predesigned questionnaire. Questionnaire was prepared following KSS for pain and function and VAS guideline. After completing the questionnaire, the individual scores were added and finally total score and mean were determined. Data were analyzed by using SPSS version 19.

Knee Society Score for pain and function Grading Points : (zero considered as a negative outcome/ no improvement) Score: 80100-Excellent, 70-79-Good, 60-69- Fair, below 60- poor. ${ }^{7}$

\section{Result}

The mean age of them was 57.7 years. Maximum study subjects of group-A (50\%) were in 61-70 years of age group and in group- $B$, nearly half $(45 \%)$ of the subjects ranged from $61-70$ years (Table I)

$\begin{array}{lcc}\begin{array}{l}\text { Table-I : Distribution of patients according to age in two groups } \\ \text { Group- } \mathbf{A}(\mathbf{n}=\mathbf{4 0})\end{array} & \text { Group-B(n=40) } \\ \begin{array}{lcc}\text { Age in years } & 8(20 \%) & 6(15 \%) \\ 41-50 & 12(30 \%) & 16(40 \%) \\ 51-60 & 20(50 \%) & 18(45 \%) \\ 61-70 & \end{array}\end{array}$

In group-A 13 (32.5\%) were male and 27 (67.5\%) were female patients. In Group-B there were $12(30 \%)$ were male and 28 (70\%) were female. (Table II)

Table-II : Distribution of patients according to sex

$\begin{array}{lcll}\text { Group- } A(n=40): & \text { Male } & 13 & 32.50 \% \\ \text { Group-B(n=40): } & \text { Female } & 27 & 67.50 \% \\ & \text { Male } & 12 & 30.0 \% \\ & \text { Female } & 28 & 70.0 \%\end{array}$

At the initial visit before injection, a mean KSS pain score of Group-A was 52.6 and Group-B was 51.3. At the 1st week, the mean KSS pain score in Group-A (65.6) was significantly higher than in Group-B (57.4). At the 1st month, mean KSS pain score also increased in both groups (72.8 and 64.4 respectively). Again at the 3rd month, mean KSS pain score still increased (81.4) in Group-A that is combination group (Fig 1). 


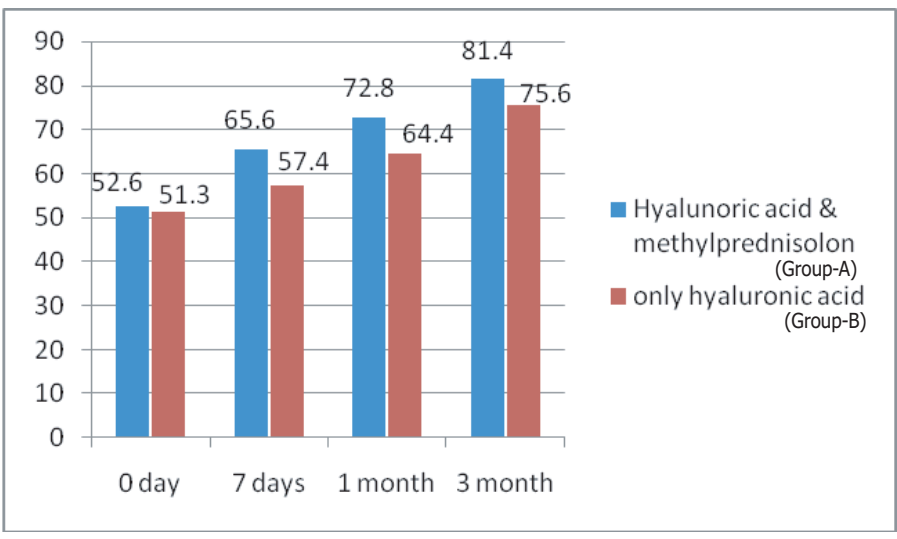

Fig-1: Comparison of pain score of KSS between two groups at different time

At the initial visit before injection, A mean KSS function score of Group-A was 46.2 and Group-B 47.9. At 1 week the mean KSS function in Group-A (61.3) was significantly higher than in Group-B (49.6). At I month, mean KSS function increased significantly in Group-A (65.1) Again at the 3rd month in combination Group (Group-A) mean KSS function score still higher (68.4) in respect of Group-B (59.4) (Fig 2).

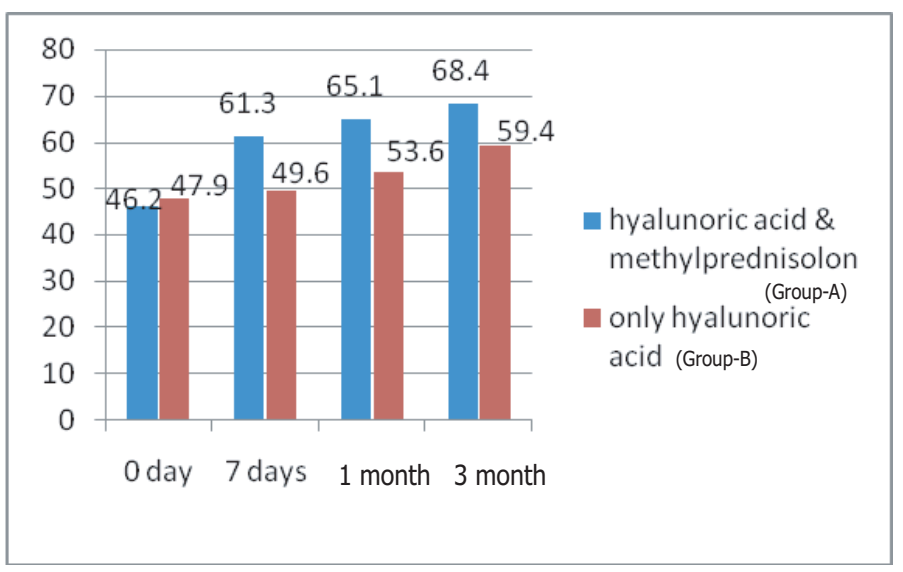

Fig-2: Comparison of function score of KSS between two groups at different time

The visual analogue scale (VAS) scores of Group-A and Group-B before injection, mean value were 5.6 and 5.4 respectively. At the 1st week it improved to 2.6 and 4.2. At the 1st month follow up it were 1.8 and 2.6 respectively and at the 3rd month the mean value was 1.4 and 2.2 .

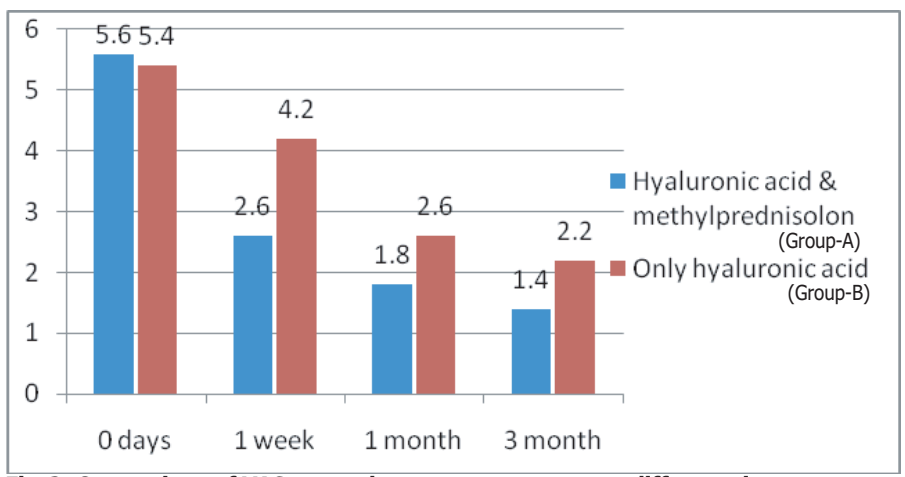

Fig-3: Comparison of VAS scores between two groups at different time

\section{Discussion}

In this study, combination of Hyaluronic acid with Methyl prednisolone was injected and its efficacy was observed. It was found that majority of cases (mean 65.6) relieved pain on 1st week by combination therapy, where as in single therapy showed less efficacy (mean 57.4).

After 1 month both the two groups' patients were evaluated and were found that combination therapy was better than single therapy. Again in 3rd month combination therapy showed sill better effect than single therapy. It was found in a study by $\mathrm{V}$ Pieterogrande that the steroid had a more rapid action, but which did not; however, last as long as that of HA. ${ }^{8}$ In our study patients got combination therapy showed early relief and long lasting improvement (in terms of decreasing pain and improving function).

A review study of meta-analysis on comparative analysis of intra-articular Hyaluronic acid versus NSAIDs, intra-articular steroids, intra-articular platelet rich plasma (PRP), or intraarticular placebo by Campbell et al (2015) ${ }^{9}$, included 14 metaanalyses with total 20,049 patients and found the highest level of evidence suggests that intra-articular Hyaluronic acid is a viable option for osteoarthritis of knee for long term relief of pain and improve function. In our study, there was rapid relief of pain and improvement of function after $1^{\text {st }}$ week as Methyl prednisolone was added along with Hyaluronic acid and effectiveness was also persists up to $3^{\text {rd }}$ month. Therefore, the effectiveness was more sustainable with intra articular combined Hyaluronic acid and Methyl prednisolone as compared to Hyaluronic acid alone at the 3rd month.

A study done by Euppayo et al in 2017 where 382 patients were evaluated for efficacy of combination therapy (Hyaluronic acid and anti-inflammatory drug) versus single Hyaluronic acid therapy. Data was taken after 1 month and 1 year. They found that, Intra-articular Hyaluronic acid and anti-inflammatory drugs had greater efficacy for pain relief than Intra-articular Hyaluronic acid alone. ${ }^{10}$ In our study, we used Methyl prednisolone with Hyaluronic acid and also found better efficacy of combination therapy in terms of pain relief and function improvement.

A randomized controlled trail was done by Thiwari $A$ and Thara A (2018) comparing the efficacy of intra-articular Hyaluronic acid versus intra-articular steroid in knee osteoarthritis. In that study the mean KSS pain and function scores and VAS were evaluated. All the three scores showed improvement at 6 months follow up. During the first month of post injection, the steroid group had better improvement in scores as compared to Hyaluronic acid group. ${ }^{11}$ We also found similar effect at 1 week in our study but in long term at 6 months follow up, they found that the results were better with Hyaluronic acid. Similar effect showed in combination therapy in the 3rd month in our study. 
Heyworth et al (2008) did a prospective, randomized clinical study comparing Hyaluronic acid, steroid, and placebo. They found that the both steroid and placebo groups showed significant pain relief for 10 weeks and its effect disappeared by 12 weeks, whereas, the patients in Hyaluronic acid group continue to have pain relief until 26 weeks. ${ }^{12}$ In our study, combination therapy showed significant pain relief within 1 st week and also at the 1st month and the 3rd month.

Askari et al (2016) compared intra-articular Hyaluronic acid with steroid in 140 cases. They found better results with intraarticular Hyaluronic acid as compared to intra-articular steroid for osteoarthritis of knee. ${ }^{13}$ In our study, with combination therapy we also found better results.

\section{Conclusion}

Considering the study result in knee osteoarthritis combination of Hyaluronic acid and Methyl prednisolone showed better efficacy to relief pain and improve function than single Hyaluronic acid infiltration. Therefore, at present this combination therapy in a single vial can be the best treatment option for knee osteoarthritis.

\section{References}

1. R.C. Lawrence, D. T. Felson, C. G. Helmick et al. "Estimates of the prevalence of arthritis and other rheumatic conditions in the United States. Part II," Arthritis and Rheumatism. vol. 58, no.1, pp. 26-35, 2008.

2. AkihiroSudo, NorikiMiyamoto, KazuhiroHorikawa, MasaoUrawa, Toru Yamakawa,TomomiYamada. Prevalence and risk factors for knee osteoarthritis in elderly Japanese men and women. Journal of Orthopaedic Science.Volume 13, Issue 5, September 2008, Pages 413-418.

3. Yavuz U, Sami S, Albayraketal A. Efficacy comparisons of the intraarticular steroid agents in the patients with knee osteoarthritis. Rheumatology International. 2012;32(11):3391-6.27.)
4. Asif S. Midterm result of cemented press Fit condylar Sigma total knee arthroplasty system. J Orthop Surg (Hong Kong). 2005 Dec; 13 (3): 280-4.

5. Goldberg VM, Buckwalter JA. Hyaluronans in the treatment of osteoarthritis of the knee: evidence for disease-modifying activity. Osteoarthritis Cartilage. 2005; 13(3):216-24.

6. Sabeena Kizhedath, Gopesh Valoth, Bindhu Vasudevan. International Journal of Basic \& Clinical Pharmacology. December 2017 | Vol 6 | Issue 12 Page 2794 - 2799.)

7. Iannitti $T$, Lodi $D$, Palmieri B. Intra-articular injections for the treatment of osteoarthritis: focus on the clinical use of Hyaluronic acid. Drugs RD. 2011; 11(1):13-27.

8. V Pieterogrande, P L Melanotte, B D' Agnolo, Michel Ulivi. Hyaluronic acid versus methylprednisolone intra-articularly injected for treatment of osteoarthritis of the knee. Current Therapeutic Research - Clinical and Experimental. 50. 691-701 https://www.researchgate.net/.../289110647_

9. Campbell KA, Erickson BJ, Saltzman BM. Is local visco supplementation injection clinically superior to other therapies in the treatment of $O A$ of the knee: a systematic review of overlapping meta-analyses. Arthroscopy. 2015; 31(10):2036-45.

10. Thippaporn Euppayo, Veerasak Punyapornwithaya, Siriwadee Chomdej, Siriwan Ongchai and Korakot Nganvongpanit. Effects of Hyaluronic acid combined with anti-inflammatory drugs compared with Hyaluronic acid alone, in clinical trials and experiments in osteoarthritis: a systematic review and meta-analysis. BMC Musculoskeletal Disorders. 2017; 18: 387.

11. Anurag Tiwari, Ankit Thora. Randomized controlled trial comparing the intraarticular Hyaluronic acid versus intra-articular steroid in osteoarthritis of knee. International Journal of Research in Orthopaedics. 2018 Jul;4(4):587590.

12. Heyworth $B E$, Lee JH, Kim PD, Lipton CB, Strauch RJ, Rosenwasser MP. Hylan versus corticosteroid versus placebo for treatment of basal joint arthritis:a prospective, randomized, double-blinded clinical trial. J Hand Surg Am. 2008; 33(1):40-8.

13. Askari A, Gholami T, NaghiZadeh MM, Farjam M, Kouhpayeh SA, Shahabfard Z. Hyaluronic acid compared with corticosteroid injections for the treatment of osteoarthritis of the knee: a randomized control trail. Springerplus. 2016;5:442. 